\title{
THE DIOPHANTINE EQUATION
}

$$
a x^{2}+2 b x y-4 a y^{2}= \pm 1
$$

\section{LIONEL BAPOUNGUÉ}

\author{
Received 30 April 2002
}

\begin{abstract}
We discuss, with the aid of arithmetical properties of the ring of the Gaussian integers, the solvability of the Diophantine equation $a x^{2}+2 b x y-4 a y^{2}= \pm 1$, where $a$ and $b$ are nonnegative integers. The discussion is relative to the solution of Pell's equation $v^{2}-\left(4 a^{2}+b^{2}\right) w^{2}=-4$.
\end{abstract}

2000 Mathematics Subject Classification: 11D09.

1. Introduction. The objective of this paper is the expansion and also the extension of [1, Section 2]. More precisely, it deals with the complete treatment of the solvability of the Diophantine equation

$$
a x^{2}+2 b x y-4 a y^{2}= \pm 1
$$

where $a$ and $b$ are positive integers. From [2, Proposition 1], (1.1) is always solvable if $a=1$. Hence, we may assume that $a>1$. Moreover, we restrict oneself to $\operatorname{gcd}(a, 2 b)=1$. In the opposite case, (1.1) is insolvable.

We denote by $\delta=4 a^{2}+b^{2}$ the discriminant of the quadratic form $a x^{2}+$ $2 b x y-4 a y^{2}$.

If $a>1, b \geq 0$, and $\operatorname{gcd}(a, 2 b)=1$, [2, Theorem 1] shows that (1.1) is insolvable if $\delta$ is a square in $\mathbb{Z}$. Hence, we will assume also that $\delta=4 a^{2}+b^{2}$ is a nonsquare in $\mathbb{Z}$, which requires $b$ to be odd. Then $\delta$ verifies $\delta \equiv 5(\bmod 8)$. Thus, (cf. [4]) the algebraic integers of $\mathbb{Q}(\sqrt{\delta})$ are the numbers $(1 / 2)(v+w \sqrt{\delta})$ with $v, w \in \mathbb{Z}$ of the same parity. Consequently, if $(1 / 2)(v+w \sqrt{\delta})$ is a unit of $\mathbb{Q}(\sqrt{\delta})$, we must have

$$
v^{2}-\delta w^{2}= \pm 4
$$

Conversely, if $(v, w)$ is an integer solution of (1.2), then $(1 / 2)(v+w \sqrt{\delta})$ is an integer of $\mathbb{Q}(\sqrt{\delta})$ (its trace is $v$ and its norm, by (1.2), is \pm 1 ) and, hence, a unit of $\mathbb{Q}(\sqrt{\delta})$. Writing $(1 / 2)\left(v_{0}+w_{0} \sqrt{\delta}\right)$ for the fundamental unit of $\mathbb{Q}(\sqrt{\delta})$, we see that the solutions in pairs of natural numbers $(v, w)$ of (1.2) comprise the values of the sequence $\left(v_{n}, w_{n}\right)(n \geq 1)$ defined by setting

$$
\frac{1}{2}\left(v_{n}+w_{n} \sqrt{\delta}\right)=\left(\frac{v_{0}+w_{0} \sqrt{\delta}}{2}\right)^{n} .
$$


Hence, we remark easily (cf. [2]) that if $(x, y)$ is a solution of (1.1), then

$$
v=-b x^{2}+8 a x y+4 b y^{2}, \quad w=x^{2}+4 y^{2}
$$

verify with $\delta=4 a^{2}+b^{2}(a>1, b \geq 1$, and $\operatorname{gcd}(a, 2 b)=1)$ the Pell's equation

$$
v^{2}-\delta w^{2}=-4 \text { with } v, w \text { odd. }
$$

Hence, our study will be based on (1.5). Thus, assuming its solvability, we give in Section 2 a necessary and sufficient condition for (1.1) to be solvable (Theorem 2.3) by methods using the arithmetic of the order $\mathbb{Z}[2 i]$ of index 2 , included in the principal ring $\mathbb{Z}+\mathbb{Z}[i]$. In the remainder of Section 2 , we establish that if (1.1) is solvable, then $\pm a$ is the norm of an element of $\mathbb{Z}[\sqrt{\delta}]$ (Proposition 2.6). Next, we prove in Section 3 that when $\delta$ is given, among all the pairs of positive coprime odd integers $(a, b)$ satisfying $\delta=4 a^{2}+b^{2}$, there is exactly one pair for which (1.1) is solvable (Theorem 3.1). That unique pair will be constructed (Theorem 4.1) in Section 4 with the aid of the following result proved in [5].

THEOREM 1.1 (Thue). If $\alpha$ and $\delta$ are integers satisfying $\delta>1, \operatorname{gcd}(\alpha, \delta)=1$, and $m$ the least integer greater than $\sqrt{\delta}$, there exist $x$ and $y$ in $] 0, m$ [ such that $\alpha y \equiv \pm x(\bmod \delta)$.

When solutions exist, we show using any of them in Section 5 that (1.1) possesses an infinity of solutions (Theorem 5.1); afterwards, we describe using it a family (Proposition 5.2). We give the conclusion of our paper in Section 6 with some numerical examples.

\section{The case $a>1, \delta$ odd nonsquare, and (1.5) solvable with $v, w$ odd}

2.1. Preliminaries. Let $v, w$ be odd integers greater than or equal to 1 such that $v^{2}-\delta w^{2}=-4$. It is clear that

$$
\operatorname{gcd}(v, w)=1
$$

because if $v$ and $w$ have a common prime factor $d$, then $d$ divides $v^{2}-\delta w^{2}=$ -4 and, therefore, $d$ divides also 2. Write (1.5) in the form

$$
\delta w^{2}=(v+2 i)(v-2 i) .
$$

The two factors of the right-hand side of (2.2) are relatively prime in $\mathbb{Z}[i]$ since any common divisor would divide $4 i$, but $w$ is odd, hence, $\operatorname{gcd}(w, 4 i)=1$. Hence, in $\mathbb{Z}[i]$, we have

$$
\operatorname{gcd}(v+2 i, v-2 i)=1
$$

Moreover, since (1.5) is written in the form (2.2), we will manipulate the elements of the nonmaximal order $\mathbb{Z}[2 i]$ of index 2 , for which we have shown 
in [3] that the half group $\mathbf{F}$ defined by

$$
\mathbf{F}=\{v+2 i \in \mathbb{Z}[2 i]: \operatorname{gcd}(N(v+2 i), 2)=1\}
$$

is factorial, where $N(\alpha)$ denotes the norm of $\alpha$. Thus, the remark of [2, Proposition 4] applied to $\mathbf{F}$ enables us to state the following definition.

DEFINITION 2.1. An odd solution $(v, w) \in \mathbb{Z}^{2}$ of (1.5) is said to be

(i) violain if, in F, $b+2 a i$ divides $v+2 i$ or $v-2 i$;

(ii) monic if, in $\mathbf{F}, b+2 a i=\operatorname{gcd}(v+2 i, \delta)$ or $\operatorname{gcd}(v-2 i, \delta)$.

Proposition 2.2. Any odd violain solution $(v, w) \in \mathbb{Z}^{2}$ of (1.5) is monic.

2.2. One criterion of solvability for (1.1). We prove the following theorem.

THEOREM 2.3. If $a \geq 3$ and $b \geq 1$ are odd integers with $\operatorname{gcd}(a, 2 b)=1$ and $\delta=4 a^{2}+b^{2}$ nonsquare in $\mathbb{Z}$, the following statements are equivalent:

(i) (1.1) has a solution $(x, y) \in \mathbb{Z}^{2}$;

(ii) (1.5) has an odd violain solution $(v, w) \in \mathbb{Z}^{2}$;

(iii) the odd minimal solution $\left(v_{0}, w_{0}\right) \in \mathbb{Z}^{2}\left(v_{0}>0, w_{0}>0\right)$ of (1.5) is monic.

Proof. (i) $\Rightarrow$ (ii). Let $(x, y) \in \mathbb{Z}^{2}$ be a solution of (1.1). We set

$$
\begin{gathered}
\varepsilon=\operatorname{sgn}\left(a x^{2}+2 b x y-4 a y^{2}\right), \\
v=\varepsilon\left(b x^{2}-8 a x y-4 b y^{2}\right), \quad w=x^{2}+4 y^{2} .
\end{gathered}
$$

As $b$ and $x$ are odd, $v$ and $w$ are also odd. Then we have

$$
v+2 i=\varepsilon\left(b x^{2}-8 a x y-4 b y^{2}\right)+2 \varepsilon\left(a x^{2}+2 b x y-4 a y^{2}\right) i
$$

so that

$$
v+2 i=\varepsilon(b+2 a i)(x+2 i y)^{2},
$$

where we see that $(v, w) \in \mathbb{Z}^{2}$ is an odd violain solution of (1.5). Further, taking the norm of the two sides of (2.7), we obtain

$$
v^{2}+4=\left(b^{2}+4 a^{2}\right)\left(x^{2}+4 y^{2}\right)^{2}=\delta w^{2}
$$

so that $(v, w)$ is an odd integer solution of (1.5).

(ii) $\Rightarrow$ (iii). Let $(v, w) \in \mathbb{Z}^{2}$ be an odd integer violain solution of (1.5). Then from equality (2.7), we have

$$
\operatorname{gcd}(v+2 i, \delta)=(b+2 a i) \operatorname{gcd}\left((x+2 i y)^{2}, b-2 a i\right) .
$$

Now, we show that

$$
\operatorname{gcd}\left((x+2 i y)^{2}, b-2 a i\right)=1 \text {. }
$$


If there exists $\alpha \in \mathbf{F}, \alpha$ is not a unit, that is, $\alpha \neq \pm 1$ such that

$$
\alpha|x+2 i y, \quad \alpha| b-2 a i
$$

then as (2.7) implies

$$
v=\varepsilon\left(b x^{2}-8 a x y-4 b y^{2}\right), \quad w=x^{2}+4 y^{2},
$$

we deduced that, in $\mathbf{F}$,

$$
\begin{gathered}
v \equiv \varepsilon\left[b(-2 i y)^{2}-8 a(-2 i y) y-4 b y^{2}\right] \\
\equiv-8 \varepsilon y^{2}(b-2 a i) \equiv 0(\bmod \alpha), \\
w \equiv 0(\bmod \alpha),
\end{gathered}
$$

that is, $\alpha$ is also a divisor of $v$ and $w$, contradicting the fact that $\operatorname{gcd}(v, w)=1$ according to (2.1). Hence, we have

$$
\operatorname{gcd}(v+2 i, \delta)=b+2 a i .
$$

Then we show that (2.14) is true for $v_{0}$ arising from the odd minimal solution of (1.5). As $(v, w)$ is an odd solution of (1.5), we have by the theory of the Pellian equation

$$
\frac{v+w \sqrt{\delta}}{2}= \begin{cases}\left(\frac{v_{0}+w_{0} \sqrt{\delta}}{2}\right)^{2 n+1}, & \text { if } v>0 \\ -\left(\frac{v_{0}-w_{0} \sqrt{\delta}}{2}\right)^{2 n+1}, & \text { if } v<0\end{cases}
$$

for some integer $n \geq 0$. Developing (2.15), we obtain

$$
4^{n} v= \begin{cases}v_{0}^{2 n+1}+\left(\begin{array}{c}
2 n+1 \\
2
\end{array}\right) v_{0}^{2 n-1} w_{0}^{2} \delta+\cdots, & \text { if } v>0 \\
-v_{0}^{2 n+1}-\left(\begin{array}{c}
2 n+1 \\
2
\end{array}\right) v_{0}^{2 n-1} w_{0}^{2} \delta-\cdots, & \text { if } v<0\end{cases}
$$

where the terms are all divisible by $\delta$ except $v_{0}^{2 n+1}$. Hence, as $v_{0}^{2} \equiv-4(\bmod \delta)$, we have

$$
v \equiv \begin{cases}(-1)^{n} v_{0}(\bmod \delta), & \text { if } v>0 \\ (-1)^{n+1} v_{0}(\bmod \delta), & \text { if } v<0\end{cases}
$$

From (2.14) and (2.17), we deduce that

$$
b+2 a i=\operatorname{gcd}(v+2 i, \delta)=\operatorname{gcd}\left( \pm v_{0}+2 i, \delta\right)=\operatorname{gcd}\left(v_{0} \pm 2 i, \delta\right)
$$

as required. This proves that $\left(v_{0}, w_{0}\right)$ is a monic solution of (1.5). 
(iii) $\Rightarrow(\mathrm{i})$. Suppose that $\left(v_{0}, w_{0}\right)$ is a monic solution of (1.5). The equality

$$
v_{0}^{2}-\delta w_{0}^{2}=-4
$$

may be expressed in the form

$$
\left(\frac{v_{0}+2 i}{b+2 a i}\right)\left(\frac{v_{0}-2 i}{b-2 a i}\right)=w_{0}^{2}
$$

where, from (2.3), $\left(v_{0}+2 i\right) /(b+2 a i)$ and $\left(v_{0}-2 i\right) /(b-2 a i)$ are coprime in $\mathbf{F}$. Hence, for some unit $\varepsilon= \pm 1$ and integers $x, y$, we have

$$
\frac{v_{0}+2 i}{b+2 a i}=\varepsilon(x+2 i y)^{2}, \quad w_{0}=x^{2}+4 y^{2} .
$$

Taking

$$
v_{0}+2 i=\varepsilon(b+2 a i)(x+2 i y)^{2}
$$

and equating coefficients of $i$ on both sides of (2.22), we obtain

$$
a x^{2}+2 b x y-4 a y^{2}=\varepsilon,
$$

showing that $(x, y) \in \mathbb{Z}^{2}$ is a solution of (1.1).

REMARK 2.4. The proof above also confirms the following result.

THEOREM 2.5. If $a \geq 3$ and $b \geq 1$ are odd integers with $\operatorname{gcd}(a, 2 b)=1$ and $\delta=4 a^{2}+b^{2}$ nonsquare in $\mathbb{Z}$, the following statements are equivalent:

(i) (1.1) has a solution $(x, y) \in \mathbb{Z}^{2}$;

(ii) (1.5) has an odd violain solution $(v, w) \in \mathbb{Z}^{2}$;

(iii) (1.5) has an odd monic solution $(v, w) \in \mathbb{Z}^{2}$.

We have also the following proposition.

Proposition 2.6. Let $a \geq 3$ and $b \geq 1$ be odd integers with $\operatorname{gcd}(a, 2 b)=1$ and $\delta=4 a^{2}+b^{2}$ nonsquare in $\mathbb{Z}$. If the Diophantine equation (1.1) has any solution $(x, y) \in \mathbb{Z}^{2}$, then

$$
a= \pm N(y \sqrt{\delta}+\mu) \quad \text { or } \quad \pm \frac{1}{4} N(x \sqrt{\delta}+\sigma), \quad \mu, \sigma \in \mathbb{Z} .
$$

In other words, $\pm a$ (resp., $\pm 4 a$ ) is the norm of an element of $\mathbb{Z}[\sqrt{\delta}]$.

Proof. We suppose that $(x, y) \in \mathbb{Z}^{2}$ is any solution of (1.1). Then the equation

$$
a t^{2}+2 b t y-4 a y^{2}-\varepsilon=0 \quad(\varepsilon= \pm 1)
$$

has an integer root, hence its discriminant is a square in $\mathbb{Z}$ :

$$
b^{2} y^{2}+4 a^{2} y^{2}-\varepsilon a=\mu^{2}, \quad \mu \in \mathbb{Z},
$$


whence

$$
\varepsilon a=y^{2}\left(b^{2}+4 a^{2}\right)-\mu^{2}=\delta y^{2}-\mu^{2}, \quad \mu \in \mathbb{Z},
$$

so that

$$
\varepsilon a=N(y \sqrt{\delta}+\mu), \quad \mu \in \mathbb{Z} .
$$

Exchanging the roles of $x$ and $y$, we obtain also

$$
4 \varepsilon a=N(x \sqrt{\delta}+\sigma), \quad \sigma \in \mathbb{Z} .
$$

3. Uniqueness of the pair $(a, b), \delta$ given. We assume in this section that $\delta$ is given and can be factorized into several sums of two squares in $\mathbb{Z}$. Then we use Theorem 2.3 to show that among all the pairs of positive integers $(a, b)$, there is exactly one pair for which (1.1) is solvable.

THEOREM 3.1. Let $\delta$ be an odd nonsquare positive integer for which (1.5) has an odd solution $(v, w) \in \mathbb{Z}^{2}$. Then among all the pairs of odd positive coprime integers $(a, b)$ satisfying $\delta=4 a^{2}+b^{2}$, there is exactly one pair $(a, b)=(A, B)$ such that (1.1) is solvable.

Proof. Let $(v, w) \in \mathbb{Z}^{2}$ be any odd violain solution of (1.5). We define positive integers $A$ and $B$ as follows:

$$
A=|a|, \quad B=b .
$$

Let $g=\operatorname{gcd}(a, b)$. Then

$$
v+2 i=g(\alpha+2 i \beta) \Longrightarrow 1=g \beta,
$$

hence $g=1$. Since $(v, w) \in \mathbb{Z}^{2}$ is an odd solution of (1.5), we have $\delta \equiv 5(\bmod 8)$, and thus $a$ and $b$ are odd. Hence, we have

$$
\operatorname{gcd}(A, B)=1
$$

with both $A$ and $B$ all odd. Then we show that $\delta=4 A^{2}+B^{2}$.

From the definition of $A$ and $B$, we see that $B+2 A i \mid v+2 i$ or $v-2 i$. Hence, we may assume, for example, that $B+2 A i \mid v+2 i$. Then (1.5) may be expressed in the form

$$
\frac{v+2 i}{B+2 A i}(v-2 i)=\frac{\delta}{B+2 A i} w^{2}
$$

where $(v+2 i) /(B+2 A i)$ and $\delta /(B+2 A i)$ are coprime elements of $\mathbf{F}$ (since $v$, $\delta$, and $B$ are odd). Equation (3.4) shows that $\delta /(B+2 A i)$ divides $v-2 i$, but 
$\delta /(B+2 A i)$ also divides $\delta$, therefore $\delta /(B+2 A i)$ divides

$$
\operatorname{gcd}(v-2 i, \delta)=B-2 A i
$$

in $\mathbf{F}$, and so

$$
\delta \mid B^{2}+4 A^{2}
$$

On the other hand, since $B+2 A i \mid \delta$, taking conjugates, we obtain $B-2 A i \mid \delta$. Let $\pi \in \mathbb{Z}[i]$ be any prime factor of $B+2 A i$ and $B-2 A i$. Then we have

$$
\frac{B^{2}+4 A^{2}}{\pi} \mid \delta
$$

Since $(v, w)$ is any odd violain solution of (1.5), we have

$$
\pi\left|\frac{v+2 i}{B+2 A i}, \quad \pi\right| \frac{v-2 i}{B-2 A i}
$$

and as $v \equiv B(\bmod 2)$, [2, Lemma 2$]$ applied to $\mathbb{Z}[i]$ shows that $\pi=1$, then the relation (3.7) becomes

$$
B^{2}+4 A^{2} \mid \delta
$$

Thus, $\delta=4 A^{2}+B^{2}$ follows from (3.6) and (3.9). Hence, $\delta=4 A^{2}+B^{2}$ is a decomposition of $\delta$ which satisfies statement (ii) of Theorem 2.3. So, by Theorem 2.3, the equation

$$
A x^{2}+2 B x y-4 A y^{2}= \pm 1
$$

is solvable. $A$ and $B$ are unique.

Applying Theorems 2.3 (or 2.5) and 3.1, we obtain the following corollary.

COROLLARY 3.2. If $\delta \equiv 5(\bmod 8)$ is a prime number for which (1.5) is solvable, $d$, e denote integers such that $\delta=4 d^{2}+e^{2}$ (they are odd, unique, and positive), then the Diophantine equation

$$
d x^{2}+2 e x y-4 d y^{2}= \pm 1
$$

is solvable.

Proof. This results from the fact that any prime number $\delta$ of the form $4 m+1$ may be represented as the sum of two squares (cf. [5]).

4. Construction of $(A, B), \delta$ given. We show in this section how the pair $(A, B)$ can be constructed. 
THEOREM 4.1. Let $\delta$ be an odd nonsquare positive integer such that (1.5) is solvable in odd integers $(v, w) \in \mathbb{Z}^{2}$. Then there exists a unique pair of coprime odd integers $(a, b)$ satisfying

$$
\begin{gathered}
b \pm a v \equiv 0(\bmod \delta), \\
0<a<\sqrt{\delta}, \quad 0<b<\sqrt{\delta}, \\
\delta=4 a^{2}+b^{2} .
\end{gathered}
$$

Then, for that unique pair $(a, b),(1.1)$ is solvable in $(x, y) \in \mathbb{Z}^{2}$.

Proof. Taking $\alpha=v$ in Theorem 1.1, we see that there exist integers $a>0$ and $b>0$ such that

$$
b \pm a v \equiv 0(\bmod \delta), \quad a<\sqrt{\delta}, b<\sqrt{\delta} .
$$

Since $(v, \delta)=1$, we have

$$
b^{2}+4 a^{2} \equiv a^{2} v^{2}+4 a^{2} \equiv-4 a^{2}+4 a^{2} \equiv 0(\bmod \delta)
$$

But $0<b^{2}+4 a^{2}<5 \delta$, hence the equations

$$
b^{2}+4 a^{2}=2 \delta, 3 \delta, 4 \delta
$$

are insolvable in $\mathbb{Z}$ since, modulus 4 , the first and the second congruences $b^{2} \equiv 2,3$ are impossible and the third imposes $b$ to be even. Hence, we have

$$
\delta=4 a^{2}+b^{2}
$$

which verifies $\delta \equiv 5(\bmod 8)$ since $(v, w) \in \mathbb{Z}^{2}$ is an odd solution of (1.5), and thus $a$ and $b$ are both odd.

Next, we show that if $(a, b)$ satisfies (4.2) and (4.5), then $\operatorname{gcd}(a, b)=1$.

Let $g=\operatorname{gcd}(a, b)$, and set $a=g a^{\prime}, b=g b^{\prime}$. Then (4.5) becomes

$$
\left(b^{\prime}\right)^{2}+4\left(a^{\prime}\right)^{2}=\delta_{1}
$$

with $\delta_{1}=\delta / g^{2}$. Relations (4.2) show that there exists $\lambda \in \mathbb{Z}$ such that $b=$ $\pm a v+\lambda \delta$, and thus $b^{\prime}= \pm a^{\prime} v+\lambda g \delta_{1}$. Replacing $b^{\prime}$ in (4.6), we obtain

$$
\left( \pm a^{\prime} v+\lambda g \delta_{1}\right)^{2}+4\left(a^{\prime}\right)^{2}=\delta_{1},
$$

and, using (1.5), we deduce from it that

$$
g\left(w^{2}\left(a^{\prime}\right)^{2} \pm 2 a^{\prime} \lambda v+\lambda^{2} g \delta_{1}\right)=1,
$$

proving that $g=1$. 
Now, we show that $(a, b)$ is unique. We suppose that $\left(a_{1}, b_{1}\right)$ is another solution of (4.2). Then from congruences

$$
b+a v \equiv b_{1}+a_{1} v \equiv 0(\bmod \delta)
$$

or

$$
b-a v \equiv b_{1}-a_{1} v \equiv 0(\bmod \delta),
$$

we see that

$$
b b_{1}+4 a a_{1} \equiv 0(\bmod \delta), \quad a b_{1}-a_{1} b \equiv 0(\bmod \delta) .
$$

From the product of the two following expressions:

$$
b^{2}+4 a^{2}=\delta, \quad b_{1}^{2}+4 a_{1}^{2}=\delta,
$$

we deduce that

$$
\left(b b_{1}+4 a a_{1}\right)^{2}+4\left(a b_{1}-a_{1} b\right)^{2}=\delta^{2}
$$

so that (dividing by $\delta^{2}$ )

$$
\left(\frac{b b_{1}+4 a a_{1}}{\delta}\right)^{2}+4\left(\frac{a b_{1}-a_{1} b}{\delta}\right)^{2}=1,
$$

which gives

$$
b b_{1}+4 a a_{1}= \pm \delta, \quad a b_{1}-a_{1} b=0 .
$$

Relations (4.15) impose

$$
\left(a_{1}, b_{1}\right)= \pm(a, b)
$$

Thus, there exists a unique solution of (4.2) satisfying $a>0, b>0$.

Finally, our last assertion is to prove that $(a, b)$ defined by (4.1) satisfies Theorem 2.3(ii). We suppose that

$$
b \pm a v \equiv 0(\bmod \delta)
$$

As $v^{2} \equiv-4(\bmod \delta)$, multiplying $(4.17)$ by $v$, we obtain

$$
b v \equiv \pm a v^{2} \equiv \pm 4 a(\bmod \delta),
$$

and so

$$
\frac{v \pm 2 i}{b+2 a i}=\frac{b v \pm 4 a}{\delta}-2\left(\frac{ \pm b+a v}{\delta}\right) i
$$


is an element of $\mathbf{F}$. Thus

$$
b+2 a i \mid v \pm 2 i .
$$

This proves that $(v, w)$ is an odd violain solution of (1.5). So, from Theorem $2.3,(1.1)$ is solvable in integers $(x, y) \in \mathbb{Z}^{2}$.

REMARK 4.2. Denoting by $\left(v_{0}, w_{0}\right)$ the odd minimal solution of (1.5), we can easily determine $A$ and $B$, such that $B+2 A i$ is the $\operatorname{gcd}\left(v_{0} \pm 2 i, \delta\right)$, using the following algorithm:

(1) (i) factorize $\delta$ in $\mathbb{Z}$;

(ii) calculate the norm of $v_{0}+2 i$ and factorize it in $\mathbb{Z}$;

(2) factorize the prime factors obtained in $\mathbf{F}$;

(3) deduce from (2) the common divisors of $\delta$ and $v_{0}+2 i$.

5. Complete set of solutions of (1.1). First of all, we prove the following theorem.

THEOREM 5.1. Under the conditions of Theorem 2.3, the Diophantine equation (1.1) has an infinity of solutions in $\mathbb{Z}$.

Proof. We assume that (1.1) has a solution $\left(x_{0}, y_{0}\right) \in \mathbb{Z}^{2}$. Then we have $\operatorname{gcd}\left(x_{0}, y_{0}\right)=1$, hence there exists $(a, b) \in \mathbb{Z}^{2}$ such that

$$
a x_{0}+b y_{0}=1
$$

We set

$$
\begin{gathered}
\left(\begin{array}{l}
x \\
y
\end{array}\right)=\left(\begin{array}{cc}
x_{0} & -\beta \\
y_{0} & \alpha
\end{array}\right)\left(\begin{array}{l}
X \\
Y
\end{array}\right), \\
g(X, Y)=f(x, y)=a x^{2}+2 b x y-4 a y^{2} .
\end{gathered}
$$

Then $g$ and $f$ are two equivalent quadratic forms. Further

$$
g(X, Y)=\varepsilon X^{2}-2 B X Y-C Y^{2}, \quad B, C \in \mathbb{Z},
$$

with

$$
\begin{aligned}
& \varepsilon=a x_{0}^{2}+2 b x_{0} y_{0}-4 a y_{0}^{2}, \\
& B=(a \beta-b \alpha) x_{0}+(b \beta+4 a \alpha) y_{0}, \\
& C=a \beta^{2}+2 b \alpha \beta+4 a \alpha^{2}
\end{aligned}
$$

But the equations

$$
f(x, y)=\varepsilon, \quad g(X, Y)=\varepsilon
$$


are equivalent, hence as

$$
g(X, Y)=\varepsilon N(X-\theta Y)
$$

where $\theta$ is a root of the equation

$$
t^{2}-\varepsilon B t+\varepsilon C=0
$$

we conclude that if $f(x, y)=\varepsilon$ has a solution in $\mathbb{Z}$, it has an infinity of solutions in $\mathbb{Z}$.

Now, we describe the family of solutions of (1.1).

Proposition 5.2. Under the conditions of Theorem 2.3, let $\left(x_{0}, y_{0}\right)$ be a particular solution of (1.1). Then the set of solutions $(x, y)$ of (1.1) is given by

$$
a x+b y+y \sqrt{\delta}= \pm\left(\frac{v_{0}+w_{0} \sqrt{\delta}}{2}\right)^{3 n}\left(a x_{0}+b y_{0}+y_{0} \sqrt{\delta}\right)
$$

in which $\left(v_{0}, w_{0}\right)$ is the minimal solution of (1.5) and $n \in \mathbb{Z}$.

Proof. Let $\left(x_{0}, y_{0}\right)$ be a particular solution of (1.1). We show how all the solutions $(x, y)$ of (1.1) may be obtained in terms of $\left(x_{0}, y_{0}\right)$ and the minimal solution $\left(v_{0}, w_{0}\right)$ of (1.5). If $(x, y)$ is any solution of (1.1) and if we set

$$
J=\frac{a x+b y+y \sqrt{\delta}}{a x_{0}+b y_{0}+y_{0} \sqrt{\delta}},
$$

the norm of $J$ is

$$
\frac{(a x+b y)^{2}-\delta y^{2}}{\left(a x_{0}+b y_{0}\right)^{2}-\delta y_{0}^{2}}=\frac{a\left(a x^{2}+2 b x y-4 a y^{2}\right)}{a\left(a x_{0}^{2}+2 b x_{0} y_{0}-4 a y_{0}^{2}\right)},
$$

that is, \pm 1 .

Moreover, $J$ is of the form $D+E \sqrt{\delta}$, where $D$ and $E$ are integers given by

$$
\begin{gathered}
D=a x x_{0}+b\left(x y_{0}+x_{0} y\right)-4 a y y_{0}, \\
E=x_{0} y-x y_{0} .
\end{gathered}
$$

Hence, by the theory of the Pellian equation, we have

$$
J= \pm\left(\frac{v_{0}+w_{0} \sqrt{\delta}}{2}\right)^{3 n}
$$

where $n \in \mathbb{Z}$. Thus, we have shown the existence of an integer $n$ such that we have (5.8).

Conversely, let $x$ and $y$ be defined by (5.8) for some $n \in \mathbb{Z}$. Taking norms of both sides of (5.8), we see that $x$ and $y$ verify (1.1). It remains to show that they are both integers. 
Define integers $M$ and $N$ by

$$
M+N \sqrt{\delta}= \pm\left(\frac{v_{0}+w_{0} \sqrt{\delta}}{2}\right)^{3 n}
$$

Then equating coefficients in (5.8), we obtain

$$
\begin{gathered}
a x+b y=M\left(a x_{0}+b y_{0}\right)+\delta N y_{0}, \\
y=M y_{0}+N\left(a x_{0}+b y_{0}\right) .
\end{gathered}
$$

Clearly, $y \in \mathbb{Z}$. Using $\delta=4 a^{2}+b^{2}$, we obtain

$$
x=(M-b N) x_{0}+4 a N y_{0}
$$

so that $x$ is also an integer.

\section{Numerical examples}

EXAMPLE 6.1. If $a=19$ and $b=71$, then $\delta=4(19)^{2}+71^{2}=6485 \equiv 5(\bmod 8)$ is nonsquare such that $\left(v_{0}, w_{0}\right)=(1369,17)$ is the minimal solution of $(1.5)$. In this case, $\operatorname{gcd}(1369+2 i, 6485)=71+38 i$ and Theorem 2.3 shows that $(1.1)$ is solvable; in fact, it is $19 x^{2}+142 x y-76 y^{2}=-1((x, y)=(1,2)$ is a solution).

EXAMPLE 6.2. If $a=3$ and $b=5$, then $\delta=4(3)^{2}+5^{2}=61 \equiv 5(\bmod 8)$ is prime such that $(v, w)=(39,5)$ is a solution of $(1.5)$; Corollary 3.2 shows that (1.1) is solvable; in fact, it is $3 x^{2}+10 x y-12 y^{2}=1((x, y)=(1,1)$ is a solution).

EXAMPLE 6.3. In case $\delta=2941=4(25)^{2}+21^{2}=4(27)^{2}+5^{2}$, we have $\delta \equiv$ $5(\bmod 8)$ nonsquare such that $\left(v_{0}, w_{0}\right)=(705,13)$ is the minimal solution of (1.5). As $\operatorname{gcd}(7054+2 i, 2941)=21-50 i$, Theorems 2.3 and 3.1 show that (1.1) is solvable only in the case when $(a, b)=(25,21)$; in fact, it is $25 x^{2}+42 x y-$ $100 y^{2}=-1((x, y)=(-3,1)$ is a solution $)$.

The equation $27 x^{2}+10 x y-108 y^{2}= \pm 1$ is insolvable.

EXAMPLE 6.4. Take $\delta=3077$. We have $\delta \equiv 5(\bmod 8)$ nonsquare such that $\left(v_{0}, w_{0}\right)=(943,17)$ is the minimal solution of $(1.5)$. The candidates for the unique pair $(a, b)$ satisfying (4.1) must be solutions of $\delta=4 a^{2}+b^{2}$. That is, $(a, b)= \pm(13,49), \pm(23,31)$. The only pair satisfying $b+a v_{0} \equiv 0(\bmod \delta)$ is $(a, b)=(13,49)$ so that $(13,49)$ is the unique pair for which (1.1) is solvable; in fact, it is $13 x^{2}+98 x y-52 y^{2}=1((x, y)=(1,2)$ is a solution).

The equation $23 x^{2}+62 x y-92 y^{2}= \pm 1$ is insolvable.

\section{REFERENCES}

[1] L. Bapoungué, Sur la résolubilité de l'équation Diophantienne $a x^{2}+2 b x y-$ $k a y^{2}= \pm 1\left[\right.$ On the solvability of the Diophantine equation $a x^{2}+2 b x y-$ 
$k a y^{2}= \pm 1$ ], C. R. Acad. Sci. Paris Sér. I Math. 309 (1989), no. 5, 235-238 (French).

[2] _ Un critère de résolution pour l'équation Diophantienne $a x^{2}+2 b x y-$ $k a y^{2}= \pm 1\left[\right.$ A criterion for the solution of the Diophantine equation $a x^{2}+$ $2 b x y-k a y^{2}= \pm 1$ ], Exposition. Math. 16 (1998), no. 3, 249-262 (French).

[3] __ Factorisation dans un ordre non maximal d'un corps quadratique [Factorization in a nonmaximal order of a quadratic field], Exposition. Math. 20 (2002), no. 1, 43-57 (French).

[4] P. Samuel, Théorie Algébrique des Nombres, Hermann, Paris, 1967 (French).

[5] D. Shanks, Solved and Unsolved Problems in Number Theory. Vol. I, Spartan Books, District of Columbia, 1962.

Lionel Bapoungué: Département de Mathématiques, École Normale Supérieure, Université de Yaoundé 1, BP 47 Yaoundé, Cameroun

E-mail address: 1bapou@uycdc.uninet.cm 


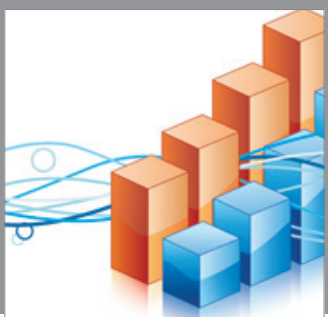

Advances in

Operations Research

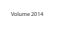

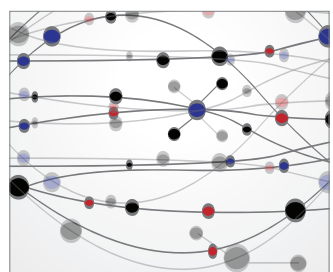

\section{The Scientific} World Journal
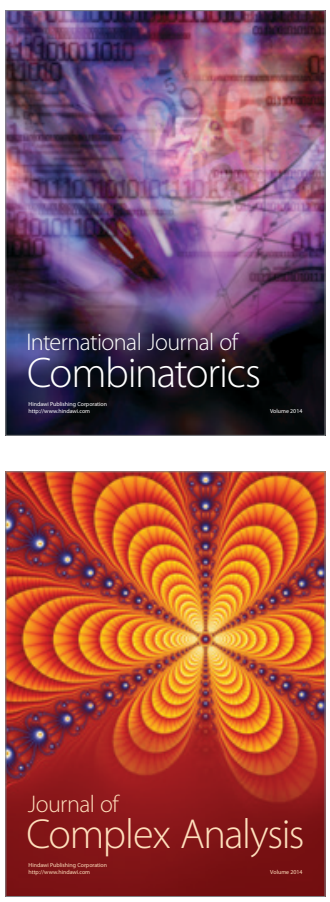

International Journal of

Mathematics and

Mathematical

Sciences
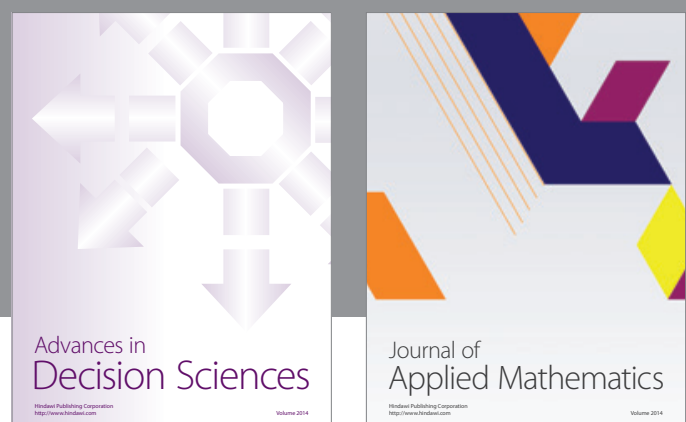

Journal of

Applied Mathematics
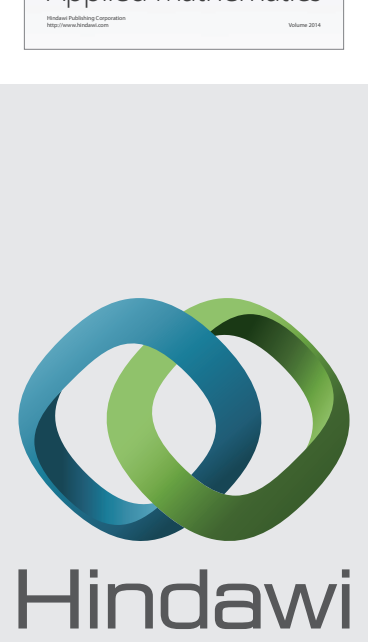

Submit your manuscripts at http://www.hindawi.com
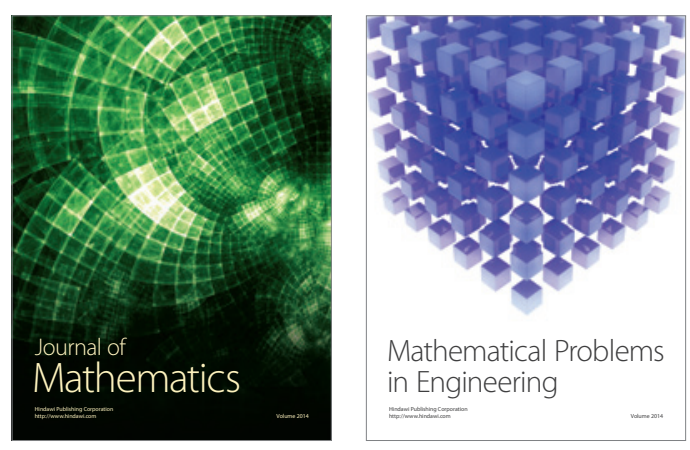

Mathematical Problems in Engineering
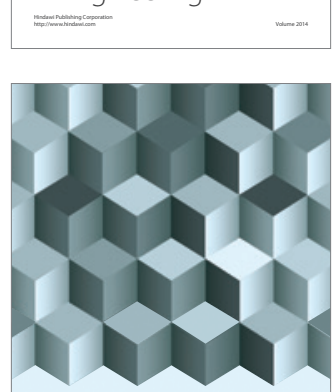

Journal of

Function Spaces
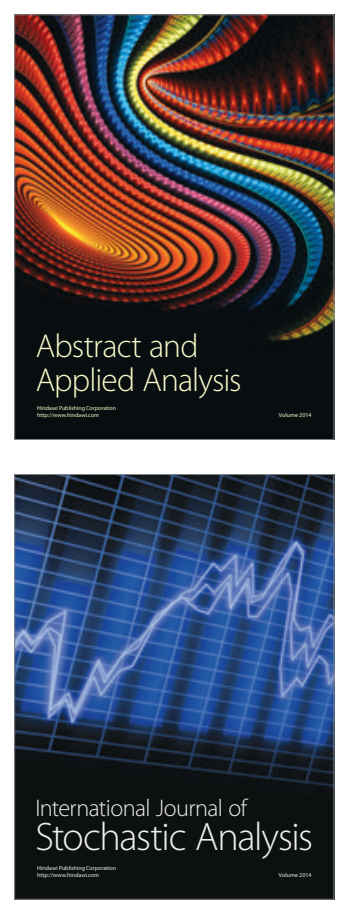

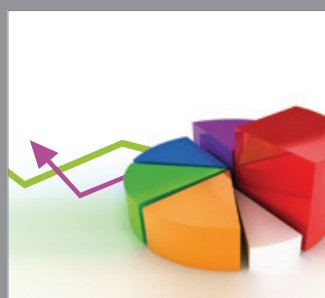

ournal of

Probability and Statistics

Promensencen
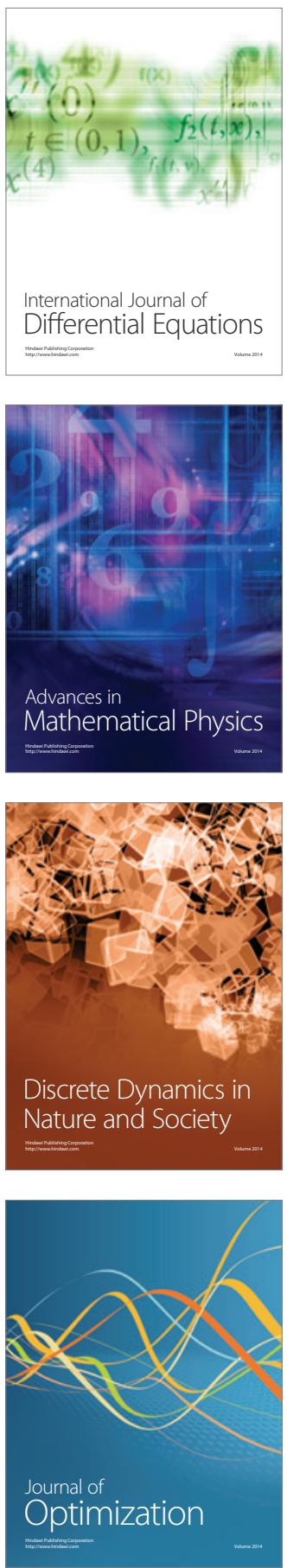